\title{
Consecuencias de la ruptura de mutualismos planta-animal para la distribución de especies vegetales en las Islas Baleares
}

\author{
Consequences of the disruption of plant-animal mutualisms for the distribution of \\ plant species in the Balearic Islands
}

ANNA TRAVESET

Institut Mediterrani d'Estudis Avançats (CSIC-UIB), C Miquel Marqués 21, 07190-Esporles, Mallorca, España; e-mail: atraveset@uib.es

\begin{abstract}
RESUMEN
Una modificación del hábitat como puede ser la introducción de especies animales exóticas que desplazan a las nativas, reduciendo sus poblaciones o conduciéndolas a la extinción, puede resultar en la ruptura de interacciones entre especies, las cuales pueden haber evolucionado durante millones de años. Los sistemas insulares son especialmente vulnerables a dichas perturbaciones, al ser normalmente menos complejos y poseer un menor número de especies que los continentales. En el presente trabajo se documentan las consecuencias que ha tenido la desaparición de la interacción planta-dispersor, después de la introducción de mamíferos carnívoros en las Islas Baleares, para las poblaciones de dos arbustos: Cneorum tricoccon L., con una distribución microareal en el Mediterráneo Occidental, y Daphne rodriguezii Texidor, endémico de la isla de Menorca. Mientras que para el primero, los carnívoros han "reemplazado" a los dispersores (lagartijas) nativos, modificando notablemente la distribución de esta especie -especialmente en Mallorca-, la ruptura del mutualismo planta-lagartija parece haber sido dramática para el segundo, reduciendo drásticamente sus poblaciones excepto en un islote donde las lagartijas aún persisten.
\end{abstract}

Palabras clave: dispersión de semillas, Cneorum tricoccon, Daphne rodriguezii, saurocoria, extinciones en islas.

\section{ABSTRACT}

The alteration of a habitat such as that caused by the introduction of exotic animal species that displace the native ones, reducing their populations or leading them to extinction, can result in the disruption of species interactions which may have evolved during millions of years. The insular ecosystems are especially vulnerable to such disturbances, as they are usually less complex and have a lower number of species than the continental ones. In the present work I document the consequences of the disruption of plant-disperser interactions, caused by the introduction of carnivorous mammals in the Balearic Islands, for the populations of two shrub species: Cneorum tricoccon L., with a distribution restricted to the western Mediterranean, and Daphne rodriguezii Texidor, endemic from Menorca island. Whereas carnivores have "replaced" the native dispersers (lizards) of the former, notably modifying its distribution -especially in Mallorca-, the plant-lizard mutualism disruption seems to have been dramatic for the second species, drastically reducing its populations except in an islet where lizards still persist.

Key words: seed dispersal, Cneorum tricoccon, Daphne rodriguezii, saurocory, insular extinctions.

\section{INTRODUCCIÓN}

Una importante consideración en biología insular es la vulnerabilidad de las islas a las perturbaciones, las cuales pueden tener notables efectos sobre la densidad de poblaciones y la distribución de muchas especies. En el caso particular de las plantas, un creciente número de estudios realizados en ecosistemas insulares (e.g., Temple 1977, Cox et al. 1991, Fujita \& Tuttle 1991, Bond 1994, Given 1995, Traveset et al. 1998, Traveset 1999, Cox \& Elmqvist 2000), o en fragmentos de bosque que actúan como islas (e.g., Spears 1987,
Jennersten 1988, Aizen \& Feisinger 1994a, 1994b, Didham et al. 1996, Kearns et al. 1998, Cunningham 2000, Parra-Tabla et al. 2000, Paton 2000), demuestra que la ruptura de una interacción ecológica (un mutualismo planta-animal, por ejemplo) puede traducirse en una reducción del éxito reproductor de la planta, e incluso conducir a la extinción de sus poblaciones. Esta ruptura es comúnmente debida a una alteración del hábitat, sea esta causada por la introducción de especies exóticas (e.g., Aizen \& Feinsinger 1994a, 1994b, Vaughton 1996, Traveset \& Riera resultados no publicados), por el cambio en el uso del suelo 
(e.g., la conversión de zonas de bosque a áreas cultivadas), por el uso de pesticidas no selectivos que afectan a la totalidad de polinizadores en el área tratada, o por la fragmentación (insularización) del hábitat (e.g., Linhart \& Feinsinger 1980, Jennersten 1988, Aizen \& Feinsinger 1994a, 1994b, Given 1995, Traveset et al. 1998, Paton 2000).

Poder predecir cual va a ser el futuro de una especie vegetal desprovista de sus animales mutualistas (uno, varios, o incluso todos), implica conocer a fondo no sólo la historia natural de esa especie (sistema sexual, conjunto de polinizadores, herbívoros y dispersores, requisitos de germinación, capacidad de reproducción vegetativa, etc.) sino también su grado de dependencia de cada una de esas especies animales y las consecuencias tanto demográficas como genéticas que conlleva su desaparición para la planta. Aunque es probable que las plantas generalistas -con varias o muchas especies de polinizadores y/o dispersores- sean menos vulnerables que las especialistas -dependientes de una o pocas especies mutualistas- (Cox et al 1991, Bond 1994, Kearns et al. 1998), tal y como se ha encontrado con algunos grupos animales (Steffan-Dewenter \& Tscharntke 2000), existe todavía poca información que evidencie una mayor probabilidad de extinción en las segundas, tanto para ecosistemas insulares como continentales. La dinámica poblacional de la planta puede verse relativamente poco afectada por cambios en la diversidad y abundancia de polinizadores y dispersores si por ejemplo el cuajado de frutos está más limitado por recursos que por polen, o si la limitación del establecimiento de plántulas es más importante que la limitación de dispersión (o sea del número de semillas depositadas en lugares adecuados) debido a efectos abióticos o bióticos (e.g., depredación de semillas por animales). De igual manera, una especie que produzca banco de semillas o que tenga capacidad de reproducirse vegetativamente, independientemente de si es especialista o generalista en sus mutualismos, tendrá una mayor probabilidad de sobrevivir, al menos durante bastante más tiempo, que una que dependa absolutamente de la producción y diseminación de sus semillas. Por otro lado, existen algunos casos documentados en los que animales introducidos han "reemplazado" a las especies nativas desaparecidas, ejerciendo su papel de mutualistas, y por tanto han evitado una extinción de la especie vegetal; tal es el caso de Zosterops en Hawai (Cox 1983) o el de carnívoros que sustituyen a las lagartijas dispersoras en las Baleares, Traveset 1995a). Lo que se desconoce todavía es en qué medida la lluvia de semillas ha sido modificada por estos "nuevos dispersores" y qué consecuencias ecológicas y evolutivas puede tener dicho reemplazo para la planta.

Según Bond (1994), para poder evaluar el riesgo de extinción de una planta desposeída de sus animales mutualistas necesitamos considerar tres factores: (1) si el mutualismo es obligado o facultativo, (2) la probabilidad de ruptura del mutualismo, y (3) la importancia de las semillas para la demografía de la planta. A su vez, hay que considerar los mecanismos que las plantas pueden evolucionar para compensar las pérdidas de sus mutualistas, como por ejemplo, (1) la capacidad de autofecundación, (2) la evolución hacia la anemogamia, o (3) la evolución de diferentes caracteres florales o de los frutos para "atraer" a un conjunto más amplio de polinizadores o dispersores, respectivamente.

En este trabajo se presentan dos casos de las Islas Baleares que muestran las consecuencias que ha tenido la ruptura del mutualismo plantadispersor para las poblaciones de plantas. En el primer caso, se trata de una especie con distribución micro-areal, restringida al Mediterráneo occidental (Cneorum tricoccon) con las mayores poblaciones en Baleares, y en el segundo, de una especie endémica de estas islas (Daphne rodriguezii), actualmente considerada en peligro de extinción por la IUCN.

\section{Las Islas Baleares}

Las Baleares están localizadas en el oeste del Mar Mediterráneo y se consideran islas para-oceánicas (Alcover et al. 1998), es decir, biológicamente similares a las oceánicas aún habiendo estado conectadas al continente en un pasado. Son islas, pues, que presentan una disharmonía importante respecto a la flora y fauna continental, con muchos grupos totalmente ausentes, y además con un número relativamente alto de especies endémicas. El archipiélago balear dista aproximadamente unos $200 \mathrm{~km}$ de la Península Ibérica y está compuesto por tres islas principales: Mallorca, Menorca y Eivissa, por dos islas más pequeñas, Formentera y Cabrera, y por un gran número de islotes. En total, la superficie insular ronda los $5.000 \mathrm{~km}^{2}$. Menorca es la isla más al norte, separada de Mallorca por unos $40 \mathrm{~km}$. La conexión con el continente tuvo lugar durante el Mesiniano (entre 5,7 y 5,35 millones de años), aunque fue a través de un desierto salino que actuó como filtro de inmigrantes (Alcover et al. 1999).

Mallorca, la mayor de las islas, consta de tres unidades geomorfológicas: la Sierra de Tramuntana, la Sierra de Levante, y las tierras bajas centrales. La Sierra de Tramuntana está 
compuesta principalmente por rocas calizas de los periodos Jurásico y Cretácico, extendiéndose unos $90 \mathrm{~km}$ a lo largo del oeste-norte de la isla. El punto más alto es el Puig Major (1.445 m), existiendo ocho picos que superan los $1.000 \mathrm{~m}$. La Sierra de Levante, en el sector sur-este, tiene una geología similar aunque con una topografía menos marcada entre 400 y $500 \mathrm{~m}$. Las tierras bajas están compuestas de un material más moderno, con cimas que no superan los $300 \mathrm{~m}$ y son principalmente terrenos de cultivo. Menorca tiene una superficie de $702 \mathrm{~km}^{2}$, con una longitud de $45 \mathrm{~km}$ y una anchura de $15 \mathrm{~km}$. Geológicamente es la más antigua de las islas y la única con material del Carbonífero. La topografía es más suave que en Mallorca, siendo su altura máxima los $358 \mathrm{~m}$ (monte El Toro), aunque presenta gargantas profundas de varios ríos que drenan la mitad sur de la isla y algunas áreas montañosas del norte. Eivissa ocupa una extensión de $541 \mathrm{~km}^{2}$, está situada a unos $100 \mathrm{~km}$ de la Península Ibérica, y tiene una topografía variada, con formaciones montañosas de 300 a $475 \mathrm{~m}$ de altura y tierras bajas cultivadas.

El clima del archipiélago balear es típicamente Mediterráneo, con temperaturas medias que oscilan entre 10 y $26^{\circ} \mathrm{C}$ y con una precipitación media anual de $600 \mathrm{~mm}$ (1970-1990). En las dos islas grandes, las especies forestales predominantes son el pino de Alepo (Pinus halepensis) y el encino (Quercus ilex). En Mallorca y en Menorca, el pinar ocupa un $22 \%$ y un $28 \%$ del territorio, respectivamente, constituyendo un $74 \%$ y un 83 \% del área forestal total. La garriga mediterránea, dominada por Olea europaea, Pistacia lentiscus, Erica arborea, Phillyrea spp., Cistus spp., representa el $13 \%$ y el $9 \%$ de estas dos islas, respectivamente, mientras que las tierras cultivadas ocupan más del $50 \%$ del área en cada una de las islas (datos del Ministerio de Agricultura, 1986, según Clevenger 1993a). En Menorca, una gran parte de estas tierras cultivadas se dedica al pastoreo de vacas. Desde que ha cesado la explotación agrícola para la producción de carbón y cal, los bosques se han recuperado y en muchos de ellos podemos encontrar una densa vegetación de sotobosque. En Eivissa, predominan los bosques de $P$. halepensis y de sabina (Juniperus phoenicea), y las especies de matorral son sobretodo P. lentiscus, Erica spp., Phillyrea spp. y Rosmarinus officinalis (Bolós \& Molinier 1969).

\section{EL CASO DE CNEORUM TRICOCCON (CNEORACEAE)}

Cneorum tricoccon L. es un arbusto perenne de talla pequeña (normalmente no supera el metro de altura, aunque algunos individuos pueden llegar a casi $2 \mathrm{~m}$; A. Traveset observación personal) que presenta una distribución disjunta en el oeste del área mediterránea (Fig. 1). Es común en Baleares y presenta poblaciones mucho más pequeñas en localidades del este y sur de la Península Ibérica, del sudeste de Francia, y en el norte de Italia (Liguria y Toscana) (Bolós 1958). Aunque este autor la cita también del sur de Cerdeña, la especie no parece encontrarse actualmente en dicha isla (I. Camarda comunicación personal). Las poblaciones más importantes de la Península Ibérica se encuentran en las provincias de Málaga y Granada, aunque la mayoría de ellas consisten en unos pocos individuos (Blanca et al. 1999). En el continente suele encontrarse bien en la maquia costera o bien en formaciones arbustivas preforestales o matorrales de degradación de las mismas. En las poblaciones del sur de la Península Ibérica, C. tricoccon vive entre 1 os 50 y $500 \mathrm{~m}$ de altitud, alcanzando los $1.000 \mathrm{~m}$ en solanas con exposición sur, y siempre sobre suelos calizos (Blanca et al. 1999). En las Islas Baleares parece haber colonizado también el interior de las islas grandes y es habitual encontrar este arbusto en el sotobosque de encinos (Quercus ilex) hasta una altitud superior a $10 \mathrm{~s} 1.000 \mathrm{~m}$.

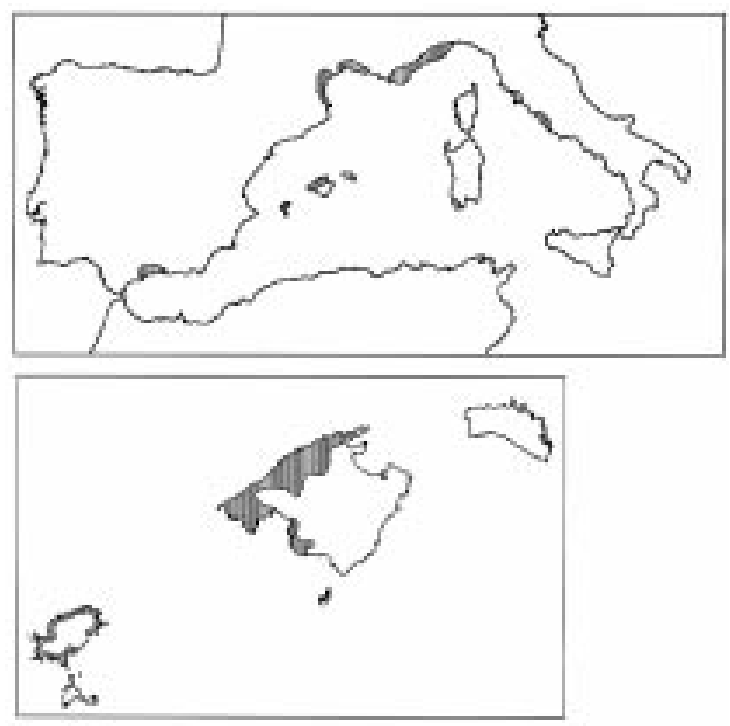

Fig. 1: Distribución de Cneorum tricoccon en el área mediterránea occidental (modificado a partir de Bolós 1958) y en las Islas Baleares (G. Alomar resultados no publicados, ligeramente modificados).

Distribution of Cneorum tricoccon in the western Mediterranean area (modified from Bolós 1958) and in the Balearic Islands (G. Alomar unpublished results, slightly modified). 
Cneorum tricoccon es una especie relictual, perteneciendo a un grupo de plantas de la flora Mediterránea que evolucionó durante el Terciario temprano bajo unas condiciones tropicales (Raven 1973, Herrera 1985). La otra especie del mismo género (que según algunos autores es sinónima de C. tricoccon, Lobreau-Callen \& Jérémie 1986) es C. trimerum, localizada en las sierras orientales de Cuba. No existe hasta el momento información sobre la ecología reproductiva de esta especie. El otro género de la familia es Neochamaelea (antes Cneorum), con una única especie, N. pulverulenta, localizada en las Islas Canarias. De ésta, únicamente se ha estudiado el sistema de dispersión (Valido 1999).

Un estudio de la estructura de edades de $C$. tricoccon en las poblaciones del sur peninsular ha revelado que hay un escaso dinamismo intrapoblacional, con un alto porcentaje $(75 \%)$ de individuos adultos de elevada edad (50-75 años), un $20 \%$ de individuos juveniles de 2 a 5 años, y un $5 \%$ aproximadamente de individuos con más de 75 años; el escaso número de plántulas, localizadas siempre bajo los adultos (Blanca et al. 1999), es indicativo de un bajo nivel de dispersión. Se desconocen quienes son sus animales dispersores en estas poblaciones, aunque observaciones preliminares apuntan a que el lagarto ocelado (Lacerta lepida Daudin 1802) puede ser uno de ellos (J. Hódar comunicación personal). En Baleares, el reclutamiento de plántulas es generalmente bajo, siendo en las poblaciones de Eivissa y Formentera donde suele encontrarse un mayor número de éstas (N. Riera comunicación personal). Tanto en las poblaciones insulares como en las continentales, se desconoce si dicho reclutamiento está más limitado por el bajo número de semillas dispersadas (o sea, por la lluvia de semillas) que por las posibilidades de establecimiento de éstas como nuevos adultos en sitios adecuados para germinar y crecer.

La floración de C. tricoccon es biestacional, tanto en las islas como en el continente. Existe una primera floración en noviembre-diciembre que se prolonga hasta febrero y otra en marzo hasta mayo. Así, en primavera es usual encontrar, en el mismo individuo, flores y frutos en distintas etapas de maduración. La fructificación y maduración de las cocas (unidades independientes de dispersión) se prolonga desde marzo hasta julio. El sistema reproductivo de Cneorum tricoccon ha sido descrito por Traveset (1995b). Se trata de una especie andromonoica, con flores funcionalmente hermafroditas y flores macho en el mismo pie. En una de las poblaciones del sur peninsular (Frigiliana, Málaga), sin embargo, se ha documentado además una baja $(<9 \%)$ presencia de flores femeninas, con estambres no funcionales (Blanca et al. 1999).
La diversidad de polinizadores es mayor en las poblaciones continentales que en las insulares. Daumann (1974) reporta al menos seis especies de abejas (Apis mellifera, Ceratina cucurbitina, $C$. cyanea, Halictus gemmens, H. smeathmanellus and Xylocopa violacea ) como principales visitantes florales de esta especie en el continente (sur de Francia). En Baleares, en cambio, la tasa de visitas por insectos es relativamente baja; mientras que en Mallorca, el principal visitante floral es Apis mellifera seguido de unas pocas especies (no identificadas) de dípteros (A. Traveset observación personal), en la isla de Cabrera no existe Apis mellifera y únicamente se han observado hormigas de pequeño tamaño de la especie Plagiolepis pygmaea (Traveset 1995b). El cuajado de frutos, sin embargo, no parece estar limitado por la cantidad de polen, ya que polinizaciones manuales no aumentan significativamente la producción de frutos (Traveset 1995b). Por otro lado, los niveles de autogamia son relativamente bajos, no superando el $10 \%$ y no se ha encontrado evidencia, al menos en las poblaciones estudiadas, de que el viento sea un vector de polinización. Tampoco se ha encontrado agamospermia en esta especie.

La producción total de frutos es muy variable entre individuos y poblaciones y está positivamente correlacionada con el tamaño de la planta (Traveset 1995b, Riera 2000). Los individuos más grandes, de más de $1 \mathrm{~m}$ de altura, no suelen encontrarse a nivel del mar sino a altitudes más bien altas y en los hábitats más húmedos (e.g., debajo de encinos), lo cual apoya la idea de que esta planta vivía su "apogeo" en las condiciones tropicales en las que evolucionó, y que por tanto ha modificado considerablemente su fisonomía con las condiciones climáticas actuales. Su reproducción es únicamente a través de semillas.

\section{Sistema de dispersión}

En la Tabla 1 se muestra una lista de las islas donde vive actualmente $C$. tricoccon y de sus dispersores en cada una de ellas. En las Pitiusas (el grupo de islas constituido por Eivissa, Formentera e islotes), los dispersores de este arbusto son exclusivamente lagartijas de la especie Podarcis pityusensis (Boscá, 1883) (Lacertidae), excepto en algunas poblaciones de Eivissa, donde hay también mamíferos carnívoros. La presencia de semillas de $C$. tricoccon en heces de estos carnívoros ha sido previamente documentada por Alcover (1984) y por Clevenger (1995). Las diferentes poblaciones de C. tricoccon en varias islas e islotes donde sus únicos dispersores son lagartijas, bien Podarcis lilfordi (Gunther, 1874) o bien P. pityusensis, re- 
TABLA 1

Dispersores de Cneorum tricoccon en las diferentes islas de las Baleares donde vive esta especie y grado de dependencia de ellos (X: débil, XX: fuerte)

Seed dispersers of Cneorum tricoccon in the different Balearic islands where this species lives and degree of dependence of them (X: weak, XX: strong)

\begin{tabular}{lcccc}
\hline Isla $\left(\mathrm{km}^{2}\right)$ & Podarcis lilfordi & Podarcis pityusensis & Martes martes & Genetta genetta \\
\hline Mallorca (3640,16) & Extinguida & - & $\mathrm{XX}$ & $\mathrm{X}$ \\
Dragonera (Mall.) $(2,88)$ & $\mathrm{XX}$ & - & - & - \\
Menorca (701,84) & Extinguida & - & $\mathrm{XX}$ & $\mathrm{X}$ \\
Cabrera (17,70) & $\mathrm{XX}$ & - & - & - \\
Illa des Conills (Cab.) $(1,37)$ & $\mathrm{XX}$ & $\mathrm{XX}$ & - & - \\
Eivissa (541,22) & - & $\mathrm{XX}$ & - & - \\
Tagomago (Eiv.) (0,625) & - & $\mathrm{XX}$ & - & - \\
Illa del Bosc (Eiv.) $(0,181)$ & - & $\mathrm{XX}$ & - & - \\
Sa Conillera (Eiv.) $(1,00)$ & - & $\mathrm{XX}$ & - & - \\
S'Espartar (Eiv.) (0,188) & - & $\mathrm{XX}$ & - & - \\
Es Vedrà (Eiv.) (0,625) & - & $\mathrm{XX}$ & - & - \\
Formentera (82,08) & - & $\mathrm{XX}$ & - \\
S'Espalmador (Form) $(1,45)$ & - & $\mathrm{XX}$ & - \\
S'Espardell (Form.) $(0,56)$ & & & - & - \\
\hline
\end{tabular}

*Datos recientes muestran la presencia de garduñas (Martes foina) (J.A. Alcover comunicación personal), supuestamente desaparecida de la isla, aunque se desconoce su abundancia

presentan un sistema ideal para determinar si estos reptiles están ejerciendo una presión de selección importante sobre el tamaño del fruto y de la semilla, tal como se ha sugerido para Neochamaelea pulverulenta en Canarias (Valido 1999). El número de semillas de Cneorum encontradas en heces de lagartijas oscila entre 1 y 6 semillas, y datos preliminares del diámetro y peso de éstas indican que, al menos en dos de las tres poblaciones estudiadas hasta ahora, las lagartijas están seleccionando los frutos de menor tamaño (Riera 2000). El tamaño de $P$. pityusensis (en particular, la longitud hocicocloaca y la anchura de la comisura bucal) varía significativamente entre poblaciones (Salvador 1984) y es por tanto de esperar, como en el caso de Canarias, que esta variación esté correlacionada con la del tamaño de los frutos. No obstante, otra presión de selección sobre el tamaño de la semilla, que puede ser contrapuesta a la que ejercen las lagartijas, es la probabilidad de germinación. Experimentos realizados con las poblaciones del sur de la Península Ibérica muestran que las semillas grandes germinan en mayor proporción que las pequeñas (Blanca et al. 1999).

\section{Ruptura del mutualismo planta-lagartija}

En Mallorca y en Menorca, debido supuestamente a la introducción masiva y relativamente reciente de mustélidos (Alcover 1980), las lagartijas se han extinguido. Algunos de estos carnívoros introducidos, martas (Martes martes L.) y ginetas (Genetta genetta (L.)) en particular, son también consumidores de frutos y resultan ser actualmente los únicos dispersores de C. tricoccon. Las consecuencias de dicha introducción, sobretodo para la lluvia de semillas de esta planta, han sido notables. En Mallorca, C. tricoccon parece haber ampliado su distribución a una mayor altitud de la que se encontraba en un pasado. Según el registro fósil, Podarcis lilfordi (endémica de Mallorca, Menorca e islotes adyacentes) no habitaba por encima de los $500 \mathrm{~m}$ (Alcover et al. 1981). Actualmente, sin embargo, es común encontrar arbustos de $C$. tricoccon a más de $1.000 \mathrm{~m}$ de altura en la Sierra de Tramuntana (Fig. 1). En Menorca, por el contrario, la desaparición de este reptil ha provocado la práctica extinción de $C$. tricoccon (actualmente con una sola y poco densa población en el sureste de la isla; O. García comunicación personal) ya que el único carnívoro consumidor de frutos existente en la isla, la marta (Martes martes spp. minoricensis), es mucho menos frugívora que su conspecífica de Mallorca (ver más abajo).

Atendiendo a la fracción de frutos de Cneorum que consumen de las plantas (44-66 \%, Traveset 1995a), los carnívoros no parecen ser, desde un punto de vista cuantitativo, tan efectivos como las lagartijas, las cuales llegan a consumir casi la totalidad de los frutos a lo largo del periodo de 
fructificación (datos de la isla de Cabrera: Traveset 1995a; datos de Pitiusas: Riera 2000). Esto puede ser debido a la mayor disponibilidad de comida (al ser muy generalistas; Clevenger 1993a,) en comparación con las lagartijas, o también a la limitada territorialidad de éstas. Desde un punto de vista cualitativo, sin embargo, al menos las martas sí podrían ser dispersores más efectivos que las lagartijas, al diseminar las semillas a mayores distancias, siempre y cuando depositen gran parte de sus heces en lugares adecuados para la germinación.

Las martas se encuentran en Mallorca y Menorca ocupando alrededor de un $50 \%$ del territorio, principalmente en pinares, encinares, en zonas de matorral y en zonas de bosque de ribera. El área de actividad de la marta oscila entre 50 y 900 ha. (Clevenger 1993b). En Mallorca, las áreas de mayor abundancia de este carnívoro son la Sierra de Tramuntana, la Sierra de Levante, y el Macizo de Randa (Clevenger 1993a). Aunque está sobretodo asociado a áreas boscosas, sus heces se han encontrado también en garrigas y en áreas abiertas, en lugares cultivados o perturbados (Clevenger 1995). Hacia los años cuarenta, sus poblaciones estuvieron a punto de extinguirse debido a una intensa caza, pero su protección legal en los últimos 30 años ha permitido su recuperación en las áreas de bosque en las dos islas. Las martas de Menorca se consideran una subespecie distinta debido a su mayor tamaño, atribuido a la falta de competencia con otros carnívoros (Alcover et al. 1986, Moreno et al. 1988, Clevenger 1993c) y a un mayor generalismo en su dieta (Clevenger 1995). Esta subespecie incluye poco material vegetal en su dieta, especialmente durante la época de fructificación de Cneorum (abril-julio), la cual coincide con una mayor explotación de aves (pollos y juveniles) y de mamíferos (Fig. 1 en Clevenger 1993c). En Mallorca, en cambio, el material vegetal (principalmente frutos) es el componente principal de la dieta de este carnívoro en todas las estaciones (Tabla 1 en Clevenger 1995). Las ginetas son también consumidoras de frutos, aunque más ocasionales que las martas. Durante primavera y otoño, el material vegetal representa una fracción importante de la dieta (Clevenger 1995). En sus heces se han encontrado también semillas de Cneorum (Alcover 1984, Clevenger 1993a, 1993c, 1995), aunque nunca de forma abundante como en el caso de las martas (Clevenger 1996, A. Traveset observación personal). El hecho de que deposita la mayor parte de las heces en letrinas, y generalmente en lugares poco adecuados para la germinación de las semillas (un $77 \%$ de las letrinas se encontraron en grietas rocosas y al borde de acantilados, expues- tas a altas temperaturas; Clevenger 1996), le hace un dispersor poco eficiente. Es, por otro lado, un importante depredador de lagartijas, especialmente en primavera y verano (Clevenger 1995), por lo que está eliminando dispersores potencialmente efectivos del hábitat. Respecto a sus ámbitos de hogar ("home range"), son supuestamente similares a los de las martas (Clevenger 1996), por lo que pueden dispersar semillas a grandes distancias.

Sólo ocasionalmente se han encontrado heces de erizos (Atelerix algirus) con semillas de Cneorum aparentemente intactas (A. Traveset observación personal). Además, las hormigas pueden ejercer una dispersión secundaria de las semillas, aunque a unas distancias presumiblemente cortas (A. Traveset observación personal).

La interacción mutualista Cneorum-lagartijas es mucho más antigua que la interacción con martas y ginetas, las cuales han sido introducidas por el hombre (Alcover 1980). Los fósiles de lagartijas más antiguos encontrados en Baleares pertenecen al Mioceno Superior (Alcover et al. 1981), aunque es probable que estos reptiles estuvieran en estas islas desde mucho antes (existen fósiles de lacértidos en la Península Ibérica que datan del Eoceno; Barbadillo observación personal). Las semillas fósiles que se han encontrado en Menorca (en diversos puntos de la isla) y en Eivissa corresponden al Plio-Pleistoceno y son del mismo tamaño y estructura de las actuales, lo que apoya la hipótesis de una constancia en el sistema de dispersión. Es posible, además, que tortugas gigantes del género Cheirogaster, cuyos fósiles han sido encontrados en Mallorca, Menorca y Eivissa (Alcover et al. 1999), actuaran también como dispersores de esta planta en un pasado. Los mamíferos autóctonos, actualmente extinguidos, que habitaron Mallorca y Menorca (aunque no Eivissa ni las otras islas) fueron una especie de musaraña (Nesiotites), un roedor (Eliomys) y un artiodáctilo con una dentición de roedor (Myotragus balearicus) (Alcover et al. 1981). Tanto la dentición de este último como los coprolitos que se han encontrado, con un material muy triturado (J.A. Alcover comunicación personal), sugieren que esta especie no pudo actuar como importante dispersor de C. tricoccon. Otros posibles dispersores de esta planta en un pasado son las aves que habitaron aquí bajo unas condiciones tropicales (Cracraft 1973). Sin embargo, el hecho de que su pariente más próximo $(N$. pulverulenta en Canarias) tampoco sea dispersado por aves, apoya la idea de que no es una planta ornitócora. Además, análisis recientes de la composición de la pulpa de los frutos muestran que 
ambas especies tienen un alto nivel de taninos (A. Traveset resultados no publicados), lo que sugiere que no pueden ser metabolizados por aves. El análisis de gran cantidad de fecas de aves actuales ha mostrado siempre la total ausencia de semillas de Cneorum, tanto en Mallorca como en Cabrera, donde se ha estudiado este aspecto más intensivamente (Traveset 1992, resultados no publicados).

Las consecuencias de la ruptura en la interacción Cneorum-lagartijas debida a la introducción de mamíferos carnívoros en Baleares han sido, en definitiva, ecológicamente importantes. La distribución de la planta en Mallorca parece haberse extendido notablemente a mayores altitudes debido a que los carnívoros introducidos son dispersores efectivos de la planta. En Menorca, en cambio, dicha ruptura ha supuesto una drástica reducción de las poblaciones de Cneorum ya que la única especie de carnívoro existente consume una baja proporción de frutos. El reemplazo de dispersores puede también haber tenido consecuencias evolutivas si los carnívoros ejercen unas presiones de selección sobre características de los frutos o de las plantas individuales diferentes de las ejercidas por lagartijas.

\section{EL CASO DE DAPHNE RODRIGUEZII (THYMELAEACEAE)}

Daphne rodriguezii es un pequeño arbusto, con ramas muy intricadas, que no sobrepasa el metro de altura. Es un paleoendemismo del litoral de la isla de Menorca (Contandriopoulos \& Cardona 1984). Actualmente, su distribución se limita a la costa nororiental de la isla, y su población más abundante se halla en el islote Colom, dentro del Parque Natural de S'Albufera, a $200 \mathrm{~m}$ de la costa. Como $C$. tricoccon, se trata también de un elemento pre-miocénico de la flora mediterránea (Raven 1973) que evolucionó bajo unas condiciones climáticas muy diferentes de las actuales y que ha sobrevivido a las glaciaciones y a los cambios drásticos de temperatura y humedad.

El hecho de encontrarlo normalmente asociado con Pistacia lentiscus y Phillyrea rodriguezii, a menudo debajo de estos arbustos, hace pensar que en este microhábitat, bien sus posibilidades de supervivencia son mayores (debido a factores biológicos o fisiológicos) o bien la lluvia de semillas dispersadas es mayor que en sitios abiertos.

Al igual que en el caso de C. tricoccon, la reproducción de $D$. rodriguezii depende exclusivamente de las semillas. Fructifica también a finales de primavera-principios de verano, y sus dispersores principales son las lagartijas endémicas (Podarcis lilfordi). No se ha observado hasta la fecha el consumo de frutos por parte de las aves. Ello puede ser debido a que estos frutos no son muy visibles ni fácilmente accesibles desde la parte superior del arbusto. Debido a la extinción de las lagartijas en la isla de Menorca, estamos de nuevo delante de otra ruptura de mutualismo, cuyas consecuencias han sido aparentemente dramáticas ya que algunas de sus poblaciones están al borde de la extinción, con un muy bajo reclutamiento de plántulas que se atribuye principalmente a una falta de dispersión más que a una limitación de establecimiento (Traveset \& Riera resultados no publicados).

El pico de floración tiene lugar a principios de primavera. La autogamia y la anemogamia en esta especie son prácticamente nulas (Traveset \& Riera resultados no publicados), al igual que se ha encontrado con otras especies del género como $D$. kamtchatica (Kikuzawa 1989) o D. gnidium (Herrera 1987). Las visitas de polinizadores son muy escasas, y en el campo sólo se han visto hormigas, escarabajos de pequeño tamaño y trips. La polinización manual, previa emasculación, muestra que el polen limita significativamente el número de semillas (Traveset \& Riera resultados no publicados). Sin embargo, los niveles de cuajado siguen siendo bajos aún añadiendo polen, lo que sugiere que puede haber además una limitación de recursos (agua, nutrientes, o ambos). Dicha baja producción de frutos parece ser común en las otras especies estudiadas del género Daphne (Herrera 1987, Kikuzawa 1989).

La pulpa de los frutos, drupas de color anaranjado, contiene un porcentaje de agua muy alto (70-86\%), lo que supone un aporte grande de este recurso para los dispersores en una época de escasez de agua en el medio. No obstante, el nivel de remoción de frutos en la planta en las poblaciones de Menorca es bajo y es normal encontrar frutos que se han secado en las ramas. La mayoría se caen debajo de la planta, aunque son rápidamente removidos por hormigas granívoras (Traveset \& Riera resultados no publicados).

La mayor densidad y abundancia de Daphne rodriguezii se encuentra en el islote Colom (más de 10.000 pies en un área de 60 ha; datos del Parque Natural de S'Albufera des Grau 1996), en la única población donde sobrevive la especie endémica de lagartija ( $P$. lilfordi sbp. balearica), también con una alta densidad de individuos $(9,875$ ind ha-1, Pérez-Mellado 1989). En este islote es frecuente encontrar heces de lagartijas, conteniendo 1-4 semillas de este arbusto, y la remoción de frutos es prácticamente del $100 \%$ (Traveset \& Riera resultados no publicados). Las semillas 
pasan intactas a través del tracto digestivo de estos reptiles, indicando por tanto que actúan como dispersores de $D$. rodriguezii.

El hecho de que la población más densa de $D$. rodriguezii se encuentra en la única área donde $P$. lilfordi está también presente y es abundante, además del hecho de que esta lagartija es el principal (y posiblemente el único) dispersor de la planta, apoya la idea de que la ruptura de la interacción planta-lagartija es lo que está limitando el crecimiento de las poblaciones en la isla de Menorca. Otras especies de lagartijas presentes en esta isla son $P$. sicula (Rafinesque, 1810) y Lacerta perspicillata Dumeril \& Bibron 1839, ambas introducidas por el hombre en tiempos relativamente recientes, aunque su dieta es principalmente insectívora (Pérez-Mellado \& Corti 1993).

\section{CONCLUSIONES}

Los dos casos documentados aquí representan ejemplos de las consecuencias potenciales de una importante perturbación, como es la introducción de especies exóticas -con la posible subsiguiente extinción de especies nativas-, en un sistema vulnerable (e.g., una isla o grupo de islas), promoviendo la ruptura de interacciones tanto ecológicas como evolutivas. La desaparición de dichas interacciones, a menudo evolucionadas durante millones de años, puede conducir a una dramática reducción de las densidades de población de uno de los participantes en la interacción, normalmente del más dependiente del otro u otros. En las rupturas de mutualismos planta-animal, son las poblaciones de plantas las que suelen sufrir cambios más drásticos, ya que éstas tienden a ser más dependientes de los animales que a la inversa. En el caso de C. tricoccon, sus "nuevos" dispersores han evitado la extinción o dramática reducción de esta planta, aunque han modificado sustancialmente su distribución en Mallorca. Por otro lado, la desaparición de la interacción planta-lagartija en la isla de Menorca está aparentemente conduciendo a la extinción de las poblaciones de $D$. rodriguezii, ya que este arbusto depende mayoritariamente de este reptil para su dispersión.

\section{AGRADECIMIENTOS}

Agradezco a todas las personas que han contribuido, en diferentes fases, a la elaboración del presente trabajo, bien ayudándome en el campo o en el laboratorio. Entre ellas destacan Antònia Sans, Núria Riera, Rodolfo Rubio, y Javier Rodríguez. También agradezco a Mari Carmen
De la Bandera y a Javier Rodríguez su eficiente ayuda prestada durante la organización del taller, y a Núria Riera el compartir sus datos, no publicados todavía, sobre Cneorum de Pitiusas. Guillem Alomar y Oscar García aportaron valiosos datos sobre la distribución en Baleares de Cneorum y Daphne, respectivamente. El presente trabajo se enmarca dentro del proyecto PB97-1174 financiado por DGICYT a la autora.

\section{LITERATURA CITADA}

AIZEN MA \& P FEINSINGER (1994a) Forest fragmentation, pollination, and plant reproduction in a Chaco dry forest, Argentina. Ecology 75: 330-351.

AIZEN MA \& P FEINSINGER (1994b) Habitat fragmentation, native insect pollinators, and feral honey bees in Argentine "Chaco Serrano". Ecological Applications 4: 378-392.

ALCOVER JA (1980) Note on the origin of the present mammalian fauna from the Balearic islands and Pityusic islands. Miscellània Zoològica (España) 6: 141-149.

ALCOVER JA (1982) Note on the origin of the present mammalian fauna from the Balearic islands and Pityusic islands. Miscellània Zoològica (España) 6: 141-149.

ALCOVER JA (1984) Uber die Nahrung der Ginsterkatze Genetta genetta (Linnaeus, 1758) auf den Inseln Mallorca, Ibiza und Cabrera. Saugetierkundliche Mitteilungen 31: 189-195.

ALCOVER JA, M DELIBES, M GOSÁLBEZ \& J NADAL (1986) Martes martes Linnaeus, 1758 a les Balears. Miscelània Zoològica (España) 10: 323-333.

ALCOVER JA, S MOYÀ-SOLẦ \& J PONS-MOYÀ (1981) Les quimeres del passat. Els vertebrats fòssils del Plio-Quaternari de les Balears i Pitiüses. Editorial Moll, Palma de Mallorca, España. 260 pp.

ALCOVER JA, R PÉREZ-OBIOL, EI YLL \& P BOVER (1999) The diet of Myotragus balearicus Bate 1909 (Artiodactila, Caprinae), an extinct bovid from the Balearic islands: evidence from coprolites. Biological Journal of the Linnean Society 66: 57-74.

ALCOVER JA, A SANS \& M PALMER (1998) The extent of extinctions of mammals on islands. Journal of Biogeography 25: 913-918.

BLANCA G, B CABEZUDO, JE HERNÁNDEZ-BERMEJO, CM HERRERA, J MOLERO MESA, J MUÑOZ \& B VALDÉS (1999) Libro rojo de la flora silvestre amenazada de Andalucía. Tomo 1: Especies en peligro de extinción. Consejería de Medio Ambiente, Sevilla, España. 302 pp.

BOLÓS O (1958) Grupos corológicos de la flora balear. Publicaciones del Instituto de Biología Aplicada 27: 49-71.

BOLÓS O \& R MOLINIER (1969) Vue d'ensemble de la végétation des îles Baléares. Vegetatio 17: 251-270.

BOND WJ (1994) Do mutualisms matter? assessing the impact of pollinator and disperser disruption on plant extinction. Philosophical Transactions of the Royal Society of London 344: 83-90. 
CLEVENGER AP (1993a) Spring and summer food habits and habitat use of the European pine marten (Martes martes) on the island of Minorca, Spain. Journal of Zoology (London) 229: 153-161.

CLEVENGER AP (1993b) Pine marten (Martes martes L.) home ranges and activity patterns on the island of Minorca, Spain. Zeitschrift für Säugetierkunde 58: 137-143.

CLEVENGER AP (1993c) The european pine marten Martes martes in the Balearic Islands, Spain. Mammal Review 23: 65-72.

CLEVENGER AP (1994) Habitat characteristics of Eurasian pine martens Martes martes in an insular Mediterranean environment. Ecography 17: 257-263.

CLEVENGER AP (1995) Seasonality and relationships of food resource use of Martes martes, Genetta genetta and Felis catus in the Balearic Islands. Revue d'Ecologie Terre et Vie 50: 109-131.

CLEVENGER AP (1996) Frugivory of Martes martes and Genetta genetta in an insular mediterranean habitat. Revue d'Ecologie Terre et Vie 51: 19-28.

CONTANDRIOPOULOS J \& MA CARDONA (1984) Caractère original de la flore endémique des Baléares. Botanica Helvetica 94: 101-131.

COX PA (1983) Extinction of the Hawaiian avifauna resulted in a change of pollinators for the ieie, Freycinetia arborea. Oikos 41: 448-454.

COX PA, T ELMQUIST, ED PIERSON \& WE RAINEY (1991) Flying foxes as strong interactors in South Pacific island ecosystems: a conservation hypothesis. Conservation Biology 5: 448-454.

COX PA \& T ELMQVIST (2000) Pollinator extinction in the Pacific islands. Conservation Biology 14: 1237. 1239.

CRACRAFT J (1973) Continental drift, paleoclimatology, and the evolution and biogeography of birds. Journal of Zoology (London) 169: 455-545.

CUNNINGHAM SA (2000) Effects of habitat fragmentation on the reproductive ecology of four plants species in Mallee woodland. Conservation Biology 14: 758-768.

DAUMANN E (1974) Zur Frage nach dem Vorkommen eines Septalnektariums bei Dycotyledonen. Zugleich ein Beitrag zur Blütenmorphologie und Bestäubungsokologie von Buxus L. und Cneorum L. Preslia, Praha 46: 97-109.

DIDHAM RK (1996) Insects in fragmented forests: a functional approach. Trends in Ecology and Evolution 11: 255-260.

FUJITA MS \& MD TUTTLE (1991) Flying foxes (Chiroptera: Pteropodidae): threatened animals of key ecological and economic importance. Conservation Biology 5: 455-463.

GIVEN DR (1995) Biological diversity and the maintenance of mutualisms. En: Vitousek PM, LL Loope \& H Adsersen (eds) Islands: biological diversity and ecosystem function: 149-162. Springer Verlag, Berlin, Alemania.

HERRERA CM (1985) Determinants of plant-animal coevolution: the case of mutualistic dispersal of seeds by vertebrates. Oikos 44: 132-141.

HERRERA J (1987) Biología reproductiva de algunas especies del matorral de Doñana. Anales del Jardín Botánico de Madrid (España) 44: 483-497.
JENNERSTEN O (1988) Pollination of Dianthus deltoides (Caryophyllaceae): effects of habitat fragmentation on visitation and seed set. Conservation Biology 2: 359-366.

KEARNS CA, DW INOUYE \& NM WASER (1998) Endangered mutualisms: the conservation of plantpollinator interactions. Annual Review of Ecology and Systematics 29: 83-112.

KIKUZAWA K (1989) Floral biology and evolution of gynodioecism in Daphne kamtchatica var. jezoensis. Oikos 56: 196-202.

LINHART YB \& P FEINSINGER (1980) Planthummingbird interactions: effects of island size and degree of specialization on pollination. Journal of Ecology 68: 745-760.

LOBREAU-CALLEN DJ \& J JEREMIE (1986) The species Cneorum tricoccon (Cneoracea, Rutales) represente a Cuba. Grana 25: 155-158.

MORENO SA, A RODRÍGUEZ \& M DELIBES (1988) Summer foods of the pine marten (Martes martes) in Majorca and Minorca, Balearic Islands. Mammalia 52: 289-291.

PARRA-TABLA V, CF VARGAS, S MAGAÑA-RUEDA \& J NAVARRO (2000) Female and male pollination success of Oncidium ascendens Lindey (Orchidaceae) in two contrasting habitat patches: forest vs agricultural field. Biological Conservation 94: 335 340.

PATON DC (2000) Disruption of bird-plant pollination systems in southern Australia. Conservation Biology 14: $1232-1234$.

PÉREZ-MELLADO V (1989) Estudio ecológico de la lagartija balear Podarcis lilfordi (Günther, 1874) en Menorca. Revista de Menorca (España) 80: 455-511.

PÉREZ-MELLADO V \& C CORTI (1993) Dietary adaptations and herbivory in lacertid lizards of the genus Podarcis from western Mediterranean islands (Reptilia: Sauria). Bonner Zoologischer Beittrage 44: 193-220.

RAVEN PH (1973) The evolution of Mediterranean floras. En: Di Castri F \& HA Mooney (eds) Mediterranean type ecosystems: origin and structure: 213-224. Springer-Verlag, Berlin, Alemania.

RIERA N (2000) Relacions mutualistes entre Podarcis pityusensis I la flora de les Pitiüses. Informe no publicado. Consejería de Fomento del Gobierno Balear, Islas Baleares, España. 10 pp.

SALVADOR A (1984) A taxonomic study of the Eivissa wall lizard, Podarcis pithyusensis Boscá 1883. En: Kuhbier H, JA Alcover \& Guerau d'Arellano Tur (eds) Biogeography and ecology of the Pityusic Islands: 393-427 Dr. W. Junk Publishers, The Hague, The Netherlands.

SPEARS EE Jr (1987) Island and mainland pollination ecology of Centrosema virginianum and Opuntia stricta. Journal of Ecology 75: 351-362.

STEFFAN-DEWENTER I \& T TSCHARNTKE (2000) Butterfly community structure in fragmented habitats. Ecology Letters 3: 449-456.

TEMPLE SA (1977) Plant-animal mutualism: coevolution with dodo leads to near extinction of plant. Science 197: 885-886. 
TRAVESET A (1992) Resultats preliminars sobre el consum de fruits per ocells a l'illa de Cabrera (Illes Balears). Anuari Ornitològic de les Balears 7: 3-9.

TRAVESET A (1995a) Seed dispersal of Cneorum tricoccon by lizards and mammals in the Balearic Archipielago. Acta Oecologica 16: 171-178.

TRAVESET A (1995b) Reproductive ecology of Cneorum tricoccon L. (Cneoraceae) in the Balearic Islands. Botanical Journal of the Linnean Society 117: 221232.

TRAVESET A (1999) La importancia de los mutualismos para la conservación de la biodiversidad en ecosistemas insulares. Revista Chilena de Historia Natural 72: 527-538.
TRAVESET A, MF WILLSON \& C SABAG (1998) Effect of nectar-robbing birds in fruit set of Fuchsia magellanicus in Tierra del Fuego. Functional Ecology 12: 459-464.

VALIDO A (1999) Ecología de la dispersión de semillas por los lagartos endémicos canarios (g. Gallotia, Lacertidae). Tesis Doctoral, Facultad de Biología, Universidad de la Laguna, Tenerife, España. 316 pp. VAUGHTON G (1996) Pollination disruption by European honeybees in the Australian bird-pollinated shrub Grevillea barklyana (Proteaceae). Plant Systematics and Evolution 200: 89-100.

Editor Asociado: R. Medel

Recibido el 30 de abril de 2001; aceptado el 8 de enero de 2002 